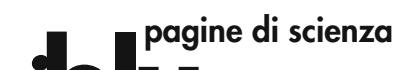 iblu
}


a cura di

Renato Betti, Angelo Guerraggio,

Settimo Termini

\section{Storie e protagonisti della matematica italiana}

per raccontare

20 anni di "Lettera Matematica Pristem"

Springer 


\section{Renato Betti, Angelo Guerraggio, Settimo Termini}

Collana i blu-pagine di scienza ideata e curata da Marina Forlizzi

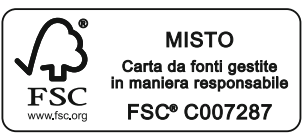

Questo libro è stampato su carta FSC amica delle foreste. Il logo FSC identifica prodotti che contengono carta proveniente da foreste gestite secondo i rigorosi standard ambientali, economici e sociali definiti dal Forest Stewardship Council

ISBN 978-88-470-2777-0

ISBN 978-88-470-2778-7 (eBook)

DOI 10.1007/978-88-470-2778-7

() Springer-Verlag Italia, 2013

Quest'opera è protetta dalla legge sul diritto d'autore e la sua riproduzione anche parziale è ammessa esclusivamente nei limiti della stessa. Tutti i diritti, in particolare i diritti di traduzione, ristampa, riutilizzo di illustrazioni, recitazione, trasmissione radiotelevisiva, riproduzione su microfilm o altri supporti, inclusione in database o software, adattamento elettronico, o con altri mezzi oggi conosciuti o sviluppati in futuro,rimangono riservati. Sono esclusi brevi stralci utilizzati a fini didattici e materiale fornito ad uso esclusivo dell'acquirente dell'opera per utilizzazione su computer. I permessi di riproduzione devono essere autorizzati da Springer e possono essere richiesti attraverso RightsLink (Copyright Clearance Center). La violazione delle norme comporta le sanzioni previste dalla legge.

Le fotocopie per uso personale possono essere effettuate nei limiti del $15 \%$ di ciascun volume dietro pagamento alla SIAE del compenso previsto dalla legge, mentre quelle per finalità di carattere professionale, economico o commerciale possono essere effettuate a seguito di specifica autorizzazione rilasciata da CLEARedi, Centro Licenze e Autorizzazioni per le Riproduzioni Editoriali, e-mail autorizzazioni@clearedi.org e sito web www.clearedi.org.

L'utilizzo in questa pubblicazione di denominazioni generiche, nomi commerciali, marchi registrati, ecc.vanche se non specificatamente identificati, non implica che tali denominazioni o marchi non siano protettivdalle relative leggi e regolamenti.

Le informazioni contenute nel libro sono da ritenersi veritiere ed esatte al momento della pubblicazione; tuttavia, gli autori, i curatori e l'editore declinano ogni responsabilità legale per qualsiasi involontario errore od omissione. L'editore non può quindi fornire alcuna garanzia circa i contenuti dell'opera.

Coordinamento editoriale: Maria Cristina Acocella

Progetto grafico e impaginazione: Ikona s.r.l., Milano

Springer-Verlag Italia S.r.l., via Decembrio 28, I-20137 Milano

Springer-Verlag fa parte di Springer Science+Business Media (www.springer.com) 


\section{Indice}

L'inizio: il 1991

Premesse e Promesse 4

Significato e limiti di “Scientia” nella cultura europea del Novecento 5

di Ludovico Geymonat

$1992 \quad 11$

Ludovico Geymonat e la rinascita della logica italiana 13

di Corrado Mangione

La Storia del pensiero filosofico e scientifico di Ludovico Geymonat $\quad 20$

di Gianni Rigamonti

$1993 \quad 23$

Una commemorazione 27

di Pier Vittorio Ceccherini

Lucio Lombardo-Radice: cultura e metodo 43

di Marta Menghini

$1994 \quad 49$

La democrazia impossibile $\quad 52$

di Piergiorgio Odifreddi

$1995 \quad 65$

Sul valore sapienziale della matematica $\quad 69$

di Ennio De Giorgi 
VI Indice

1996

Entartete Mathematik?

di Aldo Brigaglia

Una scuola senza memoria $\quad 94$

di Giovanni Prodi

1998

Metafore matematiche dell'Informatica

di Corrado Böhm

1999

No! Libertà e verità, creazione e negazione.

Palinsesto di parole e immagini

di Pietro Nastasi

2000

Torino: La storia del Centro di Studi Metodologici

di Livia Giacardi e Clara Silvia Roero

2001

Due pessimi discepoli a colloquio con un cattivo maestro

di Gianni Battimelli e Mimmo De Maria

2002

Punti critici nel tempo

di Walter Maraschini e Mauro Palma

2003

La Matematica dell'infinito

di Stefano Leonesi, Carlo Toffalori e Samanta Tordini

La fantasia e la memoria. Conversazione con Emma Castelnuovo

di Roberto Natalini e Maurizio Mattaliano 
A colloquio con Vittorio Foa

di Susanna Terracini

I ricordi della figlia. Gian Italo Bischi incontra Fulvia de Finetti

di Gian Italo Bischi

Le donne: un successo del sistema universitario italiano

278

nel Novecento

di Paola Govoni

\section{8}

Maestro di controesempi

di Umberto Bottazzini

Modellistica matematica per lo sport

di Nicola Parolini

\section{0}

Giovanni Prodi (1925-2010): un ricordo personale 